University of South Florida

DIGITAL COMMONS

Digital Commons @ University of

@ UNIVERSITY OF SOUTH FLORIDA

South Florida

Marine Science Faculty Publications

College of Marine Science

2001

\title{
Appendix: Magnetic Experiments
}

M. Fuller

Y. Touchard

C. Endris

Shipboard Scientific Party

Amelia E. Shevenell

University of California, Santa Barbara, ashevenell@usf.edu

Follow this and additional works at: https://digitalcommons.usf.edu/msc_facpub

Part of the Life Sciences Commons

\section{Scholar Commons Citation}

Fuller, M.; Touchard, Y.; Endris, C.; Shipboard Scientific Party; and Shevenell, Amelia E., "Appendix: Magnetic Experiments" (2001). Marine Science Faculty Publications. 653.

https://digitalcommons.usf.edu/msc_facpub/653

This Conference Proceeding is brought to you for free and open access by the College of Marine Science at Digital Commons @ University of South Florida. It has been accepted for inclusion in Marine Science Faculty Publications by an authorized administrator of Digital Commons @ University of South Florida. For more information, please contact digitalcommons@usf.edu. 
Exon, N.F., Kennett, J.P., Malone, M.J., et al., 2001

Proceedings of the Ocean Drilling Program, Initial Reports Volume 189

\title{
APPENDIX: MAGNETIC EXPERIMENTS'
}

\author{
M. Fuller, ${ }^{2}$ Y. Touchard, ${ }^{2}$ C. Endris, ${ }^{2}$ and \\ the Shipboard Scientific Party ${ }^{2}$
}

\section{SUMMARY}

During Leg 189, magnetic experiments were continued in an attempt to understand and mitigate the troublesome magnetic overprints encountered in the paleomagnetism of recovered Ocean Drilling Program (ODP) sediment cores. Although these overprints do not interfere with magnetostratigraphy, except at low-latitude sites, they degrade some records sufficiently that the potentially invaluable reversal and secular variation records in the cores are not usable for high-resolution work. A nonmagnetic advanced hydraulic piston core (APC) barrel assembly was run on alternate APC cores throughout the leg, and the results from cores obtained with it were compared with those cored with the standard assembly. Further comparisons were made between long-core measurements from the whole sections (i.e., before splitting) and the archive halves early during the leg. For the majority of the sites, the whole sections were used for the long-core measurements. The prevalence of disturbance at Site 1169 resulted in analysis of cores that had suffered extreme deformation. Measurement of these weak carbonate cores also makes apparent magnetic noise from sources such as core liners, which are not seen in the results from more strongly magnetized cores. Finally, we have investigated the possibility that for weak samples, such as carbonates, an instrumental malfunction can give an expression to the " 0 " declination anomaly. Preliminary results of these various investigations are presented here and will be included in a comprehensive paper to be published postcruise. 


\section{INTRODUCTION}

The problem of magnetic overprints in ODP cores has a long history, which is reviewed in Chapter 6 of the Shipboard Paleomagnetist's Handbook (Stokking et al., 1993). All paleomagnetists who have sailed on the JOIDES Resolution have encountered these problems to some degree and many have made contributions toward a solution. In the discussion that follows, credit has been given where possible, but much of the information has been handed down without full documentation, so citing references is not always possible. For a number of legs, one of the authors (M. Fuller) has been investigating the magnetic overprint problem. Unfortunately, a recurrent feature of the work has been the failure to achieve consistently repeatable results in the various tests conducted. For example, the first time the nonmagnetic cutting shoe was deployed during Leg 182, there was a remarkable improvement in the magnetic record with the almost total elimination of the " 0 " declination artifact, whereas in neighboring cores, the usual " 0 " declination was seen. Yet, later the difference between the magnetic and nonmagnetic cutting shoes was not so clear.

The two fundamental factors in the generation of overprints, which all would probably recognize, are magnetic fields and deformation. In some cases the effect of one dominates, in others both are important. Thus, when adverse drilling conditions give rise to massive deformation in the form of core "suck in" as was experienced during Leg 189 at Site 1169 , then deformation dominates and a hard vertical overprint is encountered as was also described earlier by Roberts et al. (1996). When the APC barrel with the core inside passes through the field of the bottom-hole assembly (BHA), there is no evidence of associated sediment deformation, but there is a strong magnetic field in which soft isothermal remanent magnetization (IRM) will be acquired. When moderate deformation is evident from visual inspection and measurements of anisotropy of susceptibility, or anhysteretic remanent magnetization (ARM), then the magnetization may be deflected as a passive marker in a similar way to that described by M. Okada (pers. comm., 1998). However, the configuration of the bedding planes as indicated by the maximum susceptibility plane appears to be in the form of a truncated cone with its apex upward (Herr et al., 1998). The deformation therefore gives a similar distortion of the recorded field as would a dome in a larger structural setting familiar to paleomagnetists who conduct tectonic studies. Corrections for this have been calculated by G. Acton (pers. comm., 2000).

In this paper, we describe the roles that deformation, magnetic fields, and other potentially important factors may play in the generation of overprints, as the recovered core passes through the various stages from the initial penetration of the sediment by the APC corer to final measurement with the $2 \mathrm{G}$ magnetometer. These are reviewed next and followed by the experimental work conducted during Leg 189 to investigate the various effects. 


\section{REVIEW OF POSSIBLE SOURCES OF MAGNETIC OVERPRINTS DURING RECOVERY AND MEASUREMENTS OF SEDIMENT CORES}

\section{Instrument Effects in the Measurement on the 2G Magnetometer}

It has been frequently suggested that the bias in the data toward the " 0 " declination in the ODP coordinate system (i.e., upward in the archive half, or radially inward) may be an instrument problem. In particular, it has been suggested that this is the case in weak samples, which is consistent with the familiar observation that the tray magnetization often appears to be so oriented.

One explanation that has been considered is the lack of symmetry in the half-core measurement. This was, however, investigated by J. Gee (pers. comm., 1998) and shown not be an important effect.

A second possibility, which we investigated during Leg 189 , is that the problem is caused by drift in the x-component of the direct-current superconducting quantum interference device (SQUID). This would give a bias toward the " 0 " declination direction if the drift were positive and toward the $180^{\circ}$ declination if the drift were negative. Conversely, if the principal drift were in the SQUID's y-component, then the bias would be toward the $90^{\circ}$ and $270^{\circ}$ declination, or, if in the SQUID's z-component, vertically up or down.

A related problem, which can be acute with very weak samples such as carbonates, is the contribution of magnetization of the core liner, which cannot be separated from the magnetization of the sediments in the standard measurement. A number of liners were measured as described below.

\section{Effects of the Sample Preparation on board the JOIDES Resolution}

The principal preparation that the cores are subjected to on board is core splitting. After core has been brought to the catwalk from the rig floor, it is cut into $1.5-\mathrm{m}$-long sections. It is then split into working and archive halves. For all APC cores and some extended core barrel (XCB) cores, wire splitting is used. Some XCB cores and all rotary core barrel (RCB) cores are split with a saw. It has been suggested by Witte and Kent (1988) and J. Ali (pers. comm., 2000) that the act of splitting the cores is a source of magnetic overprints. Examples of all three core types have been measured as whole-section cores before splitting and then compared with archive-half core measurements.

\section{Effects of Passage through the Bottom-Hole Assembly and Pipe from the Seafloor to the Ship}

There is a soft moment that can usually be removed from almost all ODP recovered sediments by alternating-field (AF) demagnetization to $20 \mathrm{mT}$. It has often been suggested that the magnetism of the drill pipe is responsible for this overprint. However, because the core is inside a very long magnetic pipe, this is not very likely. The core should effectively be within a magnetic shield, apart from possible leakage at the joints. Indeed, we know the fields in the pipe are not very high because 
they are measured by the Tensor tool fluxgate every time the Tensor tool is used on a piston core. The strongest fields seen are $\sim 0.1 \mathrm{mT}$.

The soft moment changes sign sometimes when the BHA is changed, which suggests that it is more likely picked up as the core passes through the BHA and, in particular, through the field of the drill bit. This suggestion was investigated during Leg 174B (Fuller and Garrett, 1998). A wash core was measured, demagnetized, remeasured, and then reassembled for tripping. The wash core was tripped in an experimental APC assembly, passing through the BHA. The experimental assembly consisted of two sections of nonmagnetic core barrel and one standard barrel above them. The standard barrel gave a relatively strong field at the joint with the nonmagnetic barrels. On its return the reassembled wash core was measured, demagnetized, and measured again. It had picked up a relatively strong moment in the same sense as the soft moment in cores drilled at the site. The field at the joint had also been recorded. The acquired magnetization was demagnetized by $20 \mathrm{mT}$, reversing the inclination to its value before tripping.

\section{Effects of Coring and Drilling}

The processes involved in piston coring and rotary drilling obviously have considerable potential to affect the magnetization of recovered sediments. These processes can be broken down into a number of different aspects for each type of coring or drilling.

In APC coring, the passage of the cutting shoe through the sediment causes deformation, as does the passage of the APC core barrels into the sediment and the core barrel's slowing to come to rest in the sediment. Earlier experiments showed that the nature of the cutting shoe can have an effect. As noted above, it was shown during Leg 182 that the use of a nonmagnetic cutting shoe markedly reduced the " 0 " declination effect on occasion. However, on other occasions, it seemed to make little difference. It appeared that the physical properties of the sediments were involved in determining the effect of the cutting shoe. During Leg 174B, it was demonstrated that the magnetization of the APC barrels was reflected in the magnetization of the sediments. This was consistent with an earlier observation that alternate cores had a similar pattern of magnetization reflecting the magnetization of the alternately used APC assemblies (Schneider et al., 1987). Our experiments suggested that the magnetization was acquired as the barrels came to rest in the sediment. We did not see the hard magnetization when the wash core was tripped, at which time it passed up and down the pipe. However, we did see this magnetization in the experimental coring, when the core barrel could have provided a field as it came to rest in the sediment. This field could then have been recorded by the sediment as it recovered from mobilization during coring. In XCB and RCB coring, there are the additional problems of the effect of the rotary cutting in the drilling process, but our main emphasis is on the APC coring, which is the method used for the sediments carrying the most recent records of the field.

In addition to magnetization processes, which are field dependent, there is also clearly the possibility of field independent deformation remagnetization. This problem has been addressed by M. Okada (pers. comm., 1998). He has developed a correction for the turning down of the sediments at the margin of the core. This was applied successfully to improve the inclination record. As noted above, this work has been extended by G. Acton (pers. comm., 2000). 


\section{EXPERIMENTAL WORK DURING LEG 189}

\section{Instrument Effects}

The noise level of the $2 \mathrm{G}$ cryogenic magnetometer has been investigated by many workers, and estimates of a moment sensitivity of $10^{-10}$ $\mathrm{Am}^{2}$ are frequently quoted. There have been numerous suggestions that with very low signals close to the noise level, results are biased toward the " 0 " declination, which corresponds to the positive $\mathrm{x}$-component in the ODP coordinate system used on the $2 \mathrm{G}$ cryogenic magnetometer.

To investigate this effect, we measured the noise level on each component for $20 \mathrm{~min}$. The plots in Figure F1 give the results for $10 \mathrm{~min}$ and reveal a small drift in the x-component, whereas the other components show noise with little drift. In each component there are fluctuations with a period of $\sim 200 \mathrm{~s}$, or 2-3 min. These drifts and noise levels are consistent with the usual performance of these magnetometers and can be mitigated by standard procedures for noise control.

In a second experiment, we measured the tray over a long period of time by moving it very slowly through the sense region. This gave us an estimate of the long-term drift, but it contains the weak signal from the tray. It was evident that the correction for the smaller drift of the $y$ - and $\mathrm{z}$-components is better than the correction for the larger drift of the $\mathrm{x}$ component.

The predominance of the drift in the $\mathrm{x}$-axis and the partial failure of a linear drift correction on this long time scale suggests that the drift correction may indeed be one explanation of the " 0 " declination results, if similar effects occur in the short time scale. However, these instrumental effects cannot be a major source of artifacts because they are so weak.

\section{Measurement of the Core Liner}

Several empty core liners were measured and produced variable results with some being magnetically quiet, whereas others had magnetic hot spots of the order $10^{-4} \mathrm{~A} / \mathrm{m}$, which is larger than the magnetization of much of the sediment (Fig. F2). Some of these hot spots were caused by attached particles and could be cleaned, but others seemed to be from the intrinsic magnetization of the plastic. Because the core is measured in these liners, no correction similar to the tray correction is possible. Such hot spots appear to give rise primarily to one- or two-point reading anomalies, so the problem was at least mitigated by our standard procedure of removing such anomalous high intensities of order $10^{-4} \mathrm{~A} / \mathrm{m}$.

\section{Comparison of Half- and Whole-Core Measurements}

Traditionally, the paleomagnetic measurement of long cores has been made on archive-half cores after the sediment core has been described. Earlier experiments during Leg 182 suggested that the process of core splitting might introduce a spurious magnetization. As noted above, there has also been the suggestion that an important aspect of the noise encountered may be a radial moment, which would, of course, cancel any measurement of the whole-round cores. Finally, unless the primary noise is in the core liner, the signal to noise ratio
F1. Noise observation for the $2 \mathrm{G}$ cryogenic magnetometer for 20 min, p. 10.

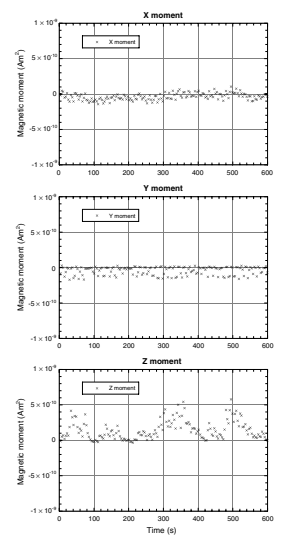

F2. Measurement of core liner magnetization, p. 11.

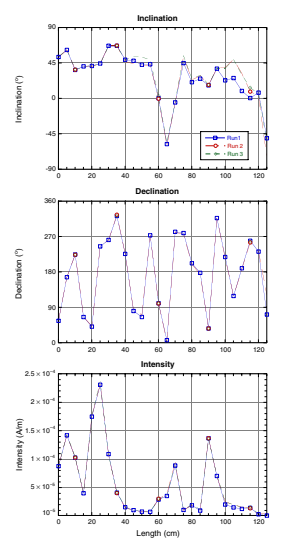


should be enhanced by a factor of 2 . For these reasons, it was decided to test the effect of the splitting during Leg 189.

The primary interest was in the APC cores, but XCB and RCB cores were also compared. At the first site, whole-round cores from Hole $1168 \mathrm{~B}$ were measured, demagnetized, and remeasured before they were split to investigate the magnetization acquired in the splitting process. The responses varied, but there was no doubt that an increase in the horizontal component of magnetization was brought about by the splitting of the core, and in some cases, this was not removed by demagnetization after splitting. Figure F3 gives a summary of the effects of splitting for inclination, declination, and intensity when the core was measured with the two halves kept together as a "whole core." Figure $\mathbf{F} 4$ shows the changes in inclination before and after the splitting and separation of the core halves. Initially, the strong drilling overprint is seen, but this is largely removed by demagnetization to $20 \mathrm{mT}$ (Fig. F4). After splitting the core, the core was reassembled and measured again as a whole-round core (Fig. F4). This plot shows that the inclination has somewhat shallowed and that two strong negative, or normal, spikes have emerged. Taking the split core apart and measuring the archive half appears to have had the effect of flattening the inclination still further (Fig. F4). Demagnetization to $20 \mathrm{mT}$ removes much of the magnetization that has shallowed the inclination, although differences between this record and the demagnetized result before the splitting remain, particularly at $\sim 100 \mathrm{~cm}$ in the section (Fig. F4). The experiments conducted with $\mathrm{XCB}$ and $\mathrm{RCB}$ cores will be analyzed postcruise. As noted above, the response to splitting the cores varies, but there is, in general, some difference between the demagnetized record before and after splitting.

\section{Observations Related to the Passage through the Bottom-Hole Assembly and Pipe from the Seafloor to the Ship}

Between Sites 1170 and 1171, a bit change was made. There was a remarkable difference in the appearance of the intensity records from the holes of the two sites, which is illustrated in Figure F5. It is natural to interpret this as a consequence of the change in bits. In this case, a moment that survives AF demagnetization has been induced in the tops of cores and affects the results to a depth of $\sim 1 \mathrm{~m}$. The drill bits were not measured in detail before use, and because both of them are now $\sim 1 \mathrm{~km}$ below the seafloor, we cannot now measure the fields involved. However, it was clear from an earlier cursory examination of the drill bits in the core technician's shop that the second drill bit type was much more magnetic than the first. This result reinforces the earlier suggestion that passage up to the ship may generate hard magnetic moments that cannot be readily demagnetized. The inclination of these same parts of the cores, which were remagnetized, was shallow. To interpret the records, we applied a filter to the data removing all values $>10^{-4} \mathrm{~A} / \mathrm{m}$.

\section{Effects of Coring: Comparison of Nonmagnetic and Standard APC Assemblies}

Throughout the leg the nonmagnetic APC core barrel assembly was used on alternate cores during APC coring. Preliminary comparisons have been made, and it is already apparent that there were no major
F3. Comparison of inclination, declination, and intensity before and after splitting Section 1891168B-3H-3, p. 12.

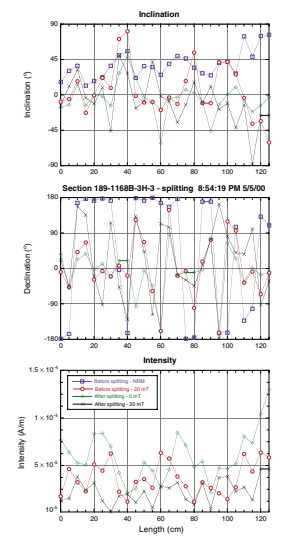

F4. Comparison of inclination before and after AF demagnetization and before and after splitting Section 189-1168A-3H-3, p. 13.

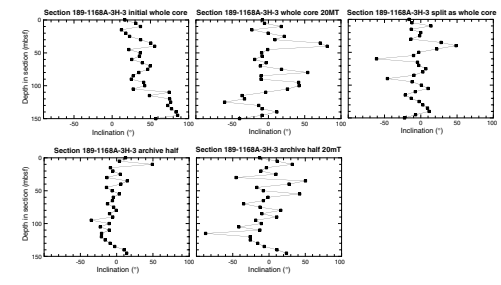

F5. Comparison of intensity records of APC cores, Sites 1170 and 1171, p. 14.

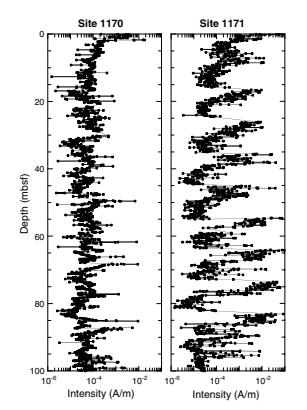


differences between cores with the different assemblies. As an example of the small differences seen in many cores, Figure F6 shows the inclination and intensity for Cores $189-1170 \mathrm{~B}-4 \mathrm{H}, 5 \mathrm{H}, 6 \mathrm{H}$, and $7 \mathrm{H}$. After AF demagnetization, both sets of core have very similar intensities and both appear to record similar inclinations. Although there were some larger differences in other cores, the overall impression is that the nonmagnetic APC core barrel does not make a substantial difference in these cores. A more detailed analysis will be made postcruise using discrete samples to constrain core orientation.

\section{Conclusions and Preliminary Recommendations}

The results obtained during Leg 189 have demonstrated the following:

1. Instrument effects may explain the occurrence of a bias toward the " 0 " declination in long-core measurements of very weak sediments, if the X-component SQUID has a higher drift than the y-component SQUID and if the drift is not properly corrected.

2. Core liners can give rise to anomalous magnetization. This can be recognized by the one- or two-point anomalies that are produced. Fortunately, in very weak sediments the anomalies are sufficiently distinctive that they can be removed from the final data by rejecting all measurements with an intensity greater than some chosen upper limit for the sediment magnetization. This also eliminates the high intensities found at the top of many cores.

3. The process of core splitting by both wire and saw can introduce magnetic noise. It is therefore recommended that the paleomagnetic measurements on the long cores be made before splitting to avoid the possibility of such magnetic noise. This would give a factor of 2 increase in the material being measured in the sense region of the coils. Providing that the principal noise is not in the core liner, which would, of course, also be increased by a factor of 2, this could provide a significant enhancement of signal to noise. If there are important radial components of noise, their effect will be canceled. The change in core flow through the laboratory proved to be an improvement.

4. A soft IRM can be acquired as the recovered core passes through the field of the drill string and, in particular, at the field of the drill bit. This IRM is removed by routine demagnetization. Whether a harder moment can also be acquired in the piston cores, because the sediment may be in a partially fluidized state, is not clear, but we have not found evidence of it.

5. The standard cutting shoe has a strong magnetic field at its cutting surface that can affect the magnetization of the sediment. It is recommended that nonmagnetic cutting shoes be used. This will, on some occasions, improve the paleomagnetic record.

6. The existence of hot spots in the APC barrels can be recorded in the magnetization of the sediments, as was demonstrated with the mixed APC assembly of standard and nonmagnetic core barrels during Leg 182. However, it is not clear that the use of solely nonmagnetic APC core barrel assemblies is going to make a significant improvement in the paleomagnetic record.
F6. Comparison of inclination and intensity of cores taken with standard and nonmagnetic assemblies, p. 15.

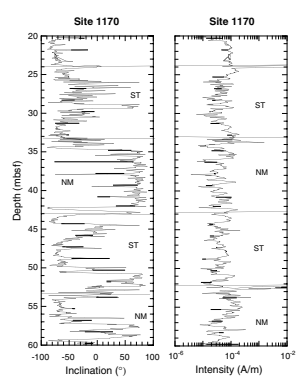


M. FULLER ET AL.

APPENDIX: MAgnetic EXPERIMENTS

A frustrating aspect of this work has been the inconsistency of the results obtained from essentially similar experiments (e.g., the effect of core splitting and the effect of the nonmagnetic cutting shoe). This inconsistency is obviously because we do not yet understand the relevant phenomena well enough to design our experiments correctly. However, the preliminary recommendations made should significantly help.

\section{ACKNOWLEDGMENTS}

The authors are very grateful to all the members of the JOIDES Resolution crew who have cheerfully assisted in this work. In particular, we thank Ron Grout, Joe Attryde, and Sam McClelland for answering our many questions and helping us understand some of the mysteries of their work. 


\section{REFERENCES}

Fuller, M., and Garrett, E., 1998. Site 395 appendix: magnetic experiments. In Becker, K., Malone, M.J., et al., Proc. ODP, Init. Repts. 174B, 47-49 [CD-ROM]. Available from: Ocean Drilling Program, Texas A\&M University, College Station, TX 778459547, U.S.A.

Herr, B., Fuller, M., Haag, M., and Heider, F., 1998. The influence of drilling on two records of the Matuyama-Brunhes polarity transition in marine sediment cores near Gran Canaria. In Weaver, P.P.E., Schmincke, H.-U., Firth, J.V., and Duffield, W. (Eds.), Proc. ODP, Sci. Results, 157: College Station, TX (Ocean Drilling Program), 57-69.

Roberts, A.P., Stoner, J.S., and Richter, C., 1996. Coring-induced magnetic overprints and limitations of the long-core paleomagnetic measurement technique: some observations from Leg 160, eastern Mediterranean Sea. In Emeis, K.-C., Robertson, A.H.F., Richter, C., et al., Proc. ODP, Init. Repts., 160: College Station, TX (Ocean Drilling Program), 497-505.

Schneider, D.A., and Van Damme, D., and Shipboard Scientific Party, 1987. Paleomagnetism of Site 709. In Backman, J., Duncan, R.A., Peterson, L.C., et al., Proc. ODP, Init. Repts., 115: College Station, TX (Ocean Drilling Program), 474-478.

Stokking, L.B., Musgrave R.J., Bontempo, D., and Autio, W., 1993. Handbook for Shipboard Paleomagnetists. ODP Tech. Note, 18: College Station, TX (Ocean Drilling Program).

Witte, W.K., and Kent, D.V., 1988. Revised magnetostratigraphies confirm low sedimentation rates in Arctic ocean cores. Quat. Res., 29:43-53. 
M. FULLER ET AL.

APPENDIX: MAgnetic EXPERIMENTS

Figure F1. Noise observation for the $2 \mathrm{G}$ cryogenic magnetometer for $20 \mathrm{~min}$.
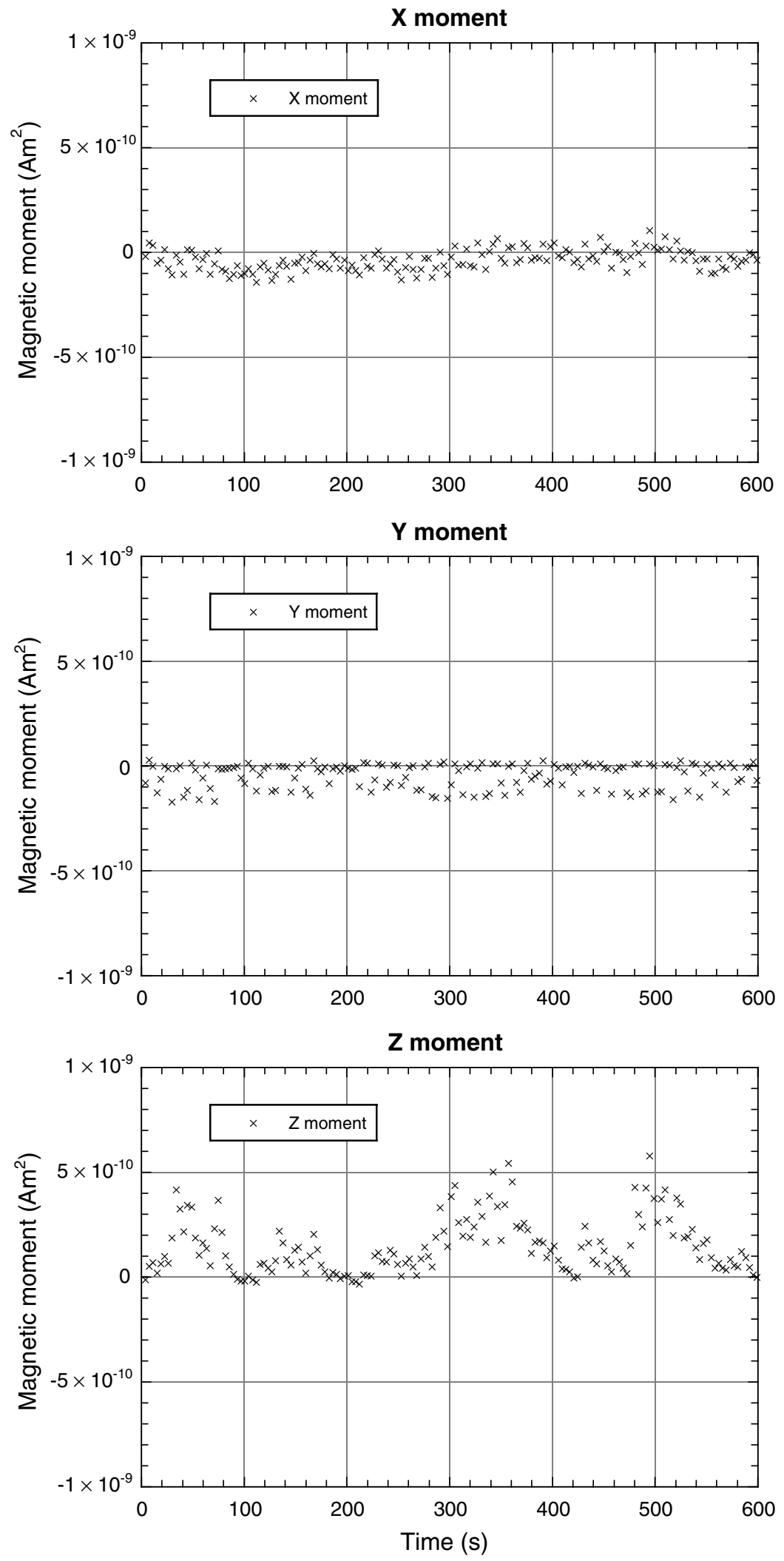
M. FULLER ET AL.

ApPendix: MAgnetic EXPERIMENTS

Figure F2. Measurement of core liner magnetization.
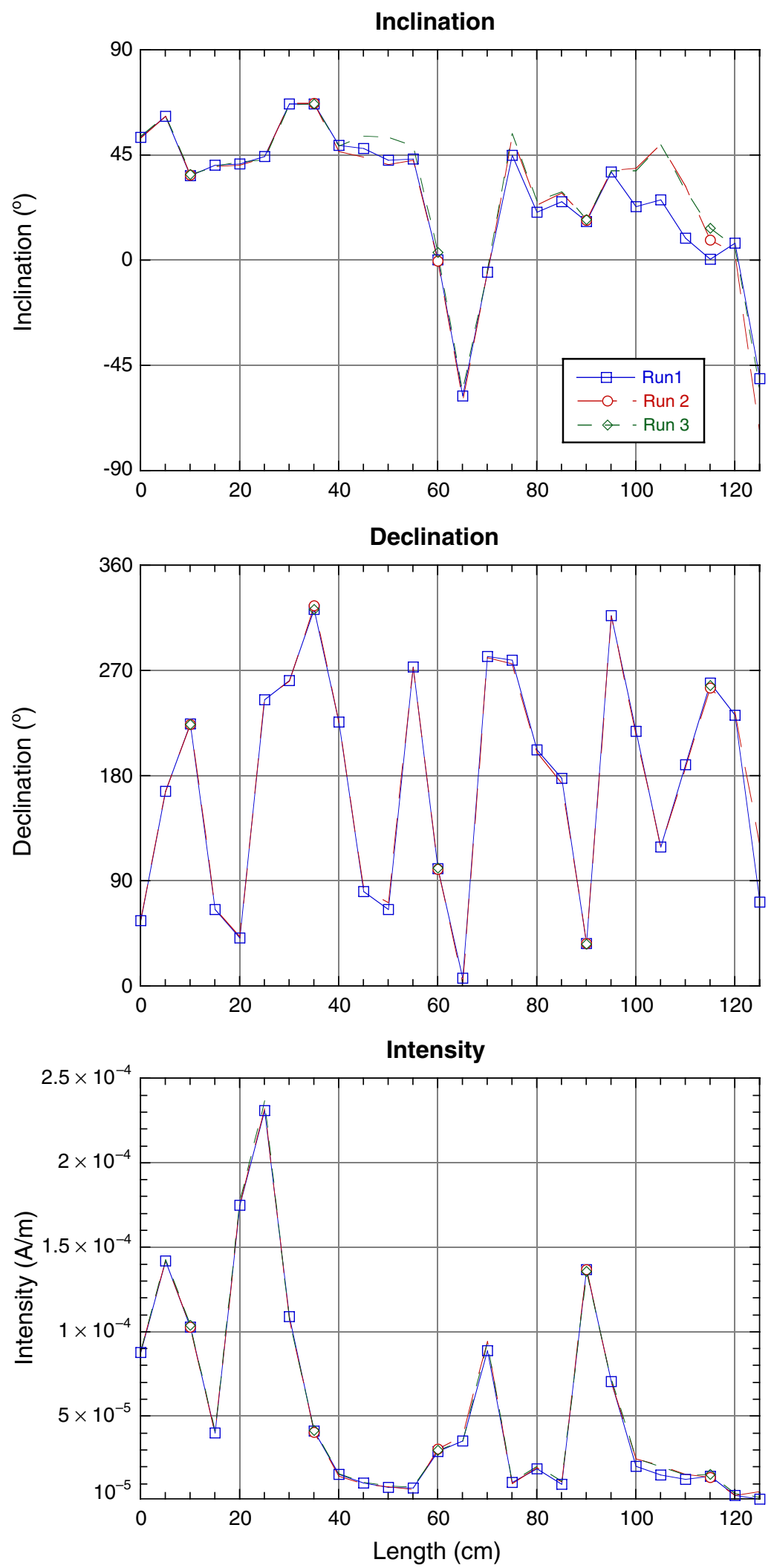
M. Fuller et AL.

APPENDiX: MAgnetic EXPERIMENTS

Figure F3. Comparison of inclination, declination, and intensity before and after splitting Section 189 1168B-3H-3.
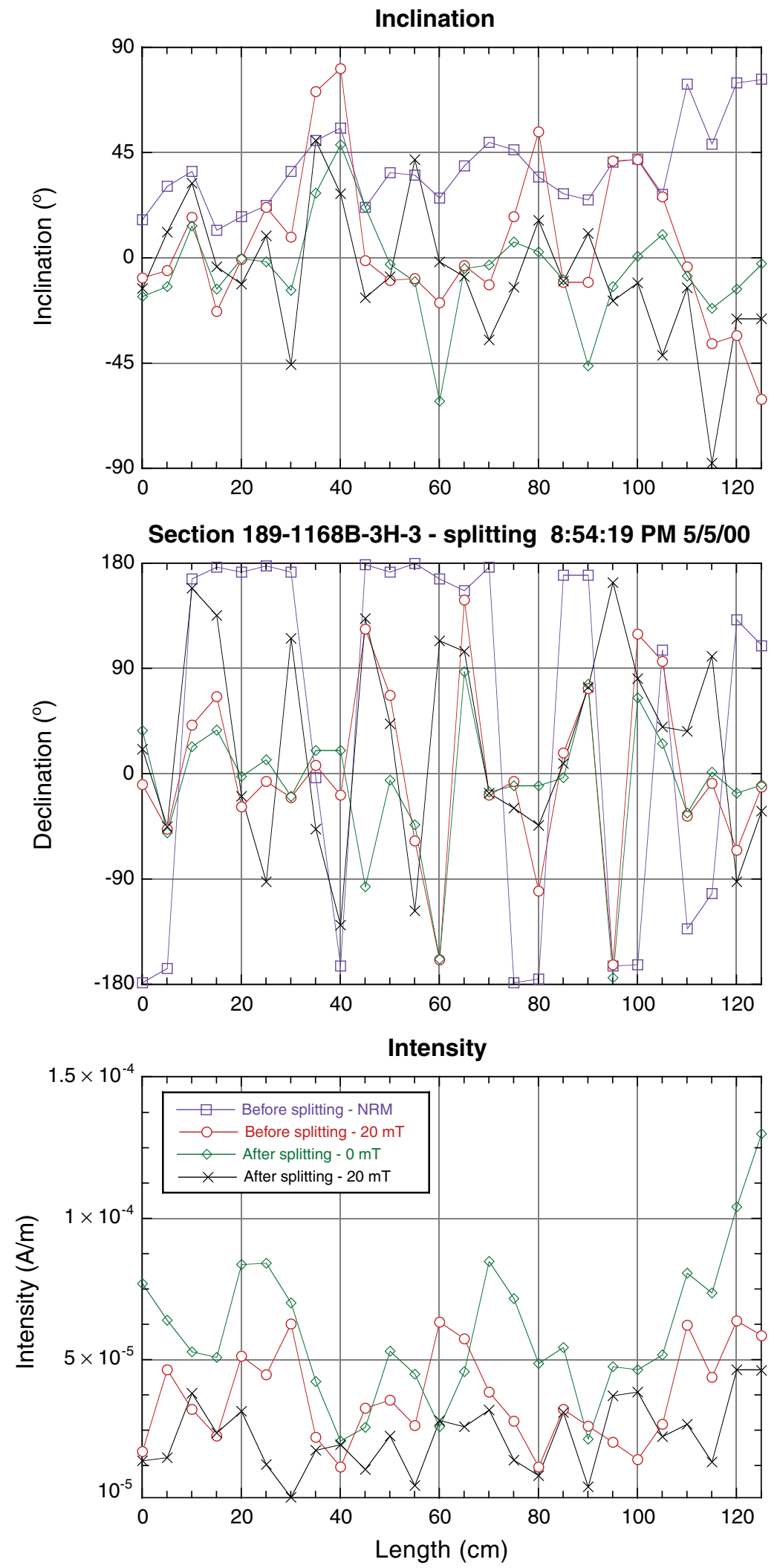
Figure F4. Comparison of inclination before and after AF demagnetization and before and after splitting Section 189-1168A-3H-3.
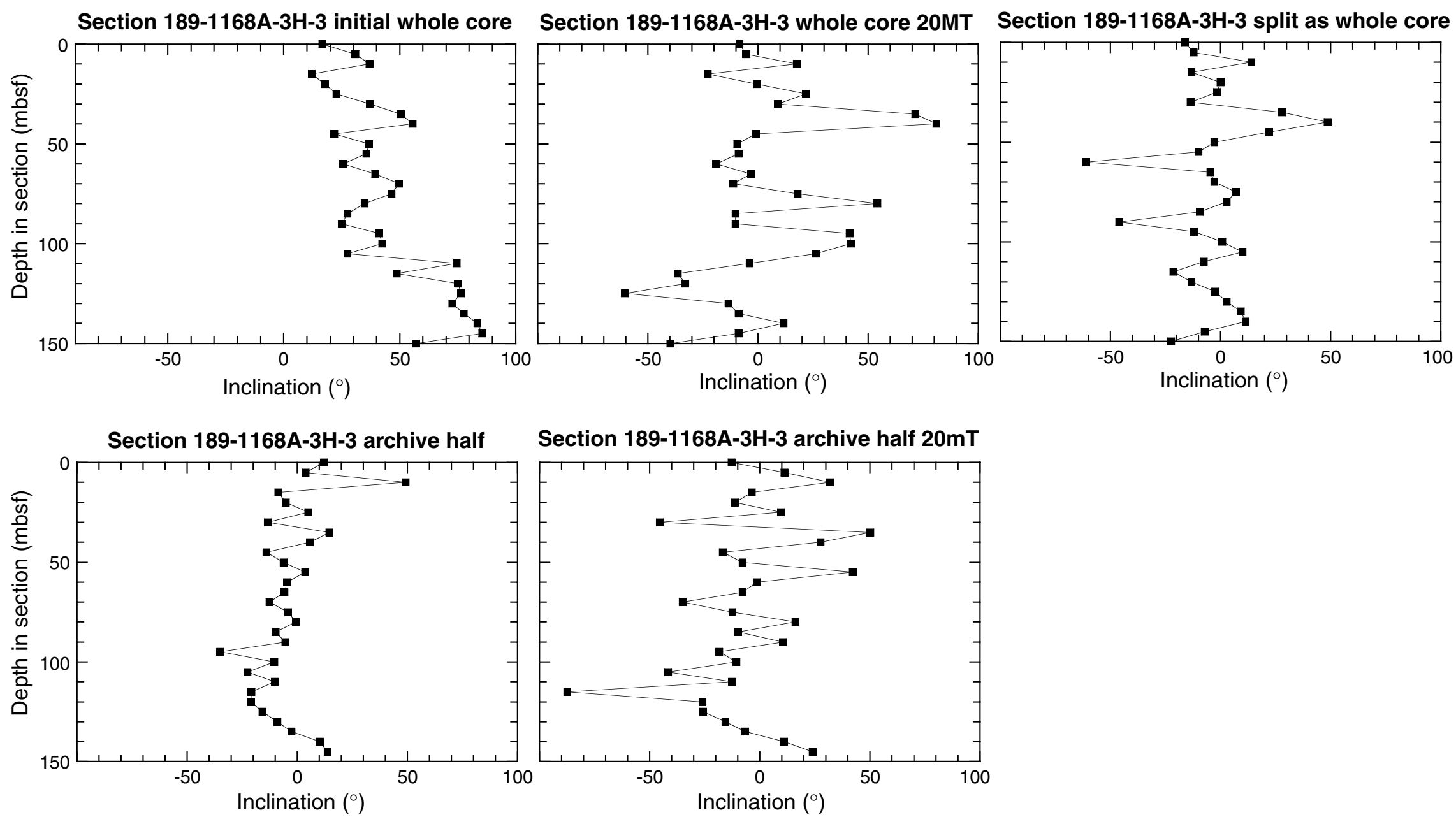

Section 189-1168A-3H-3 archive half 20mT

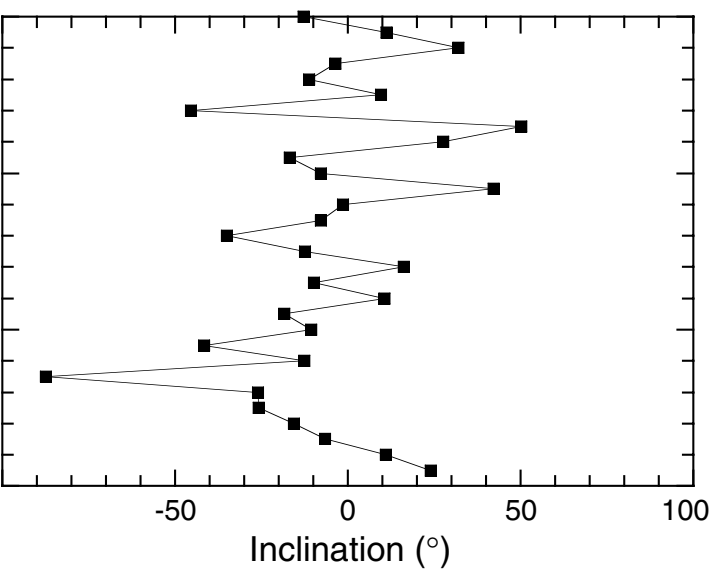


M. Fuller et AL.

Figure F5. Comparison of intensity records of APC cores from Sites 1170 and 1171.

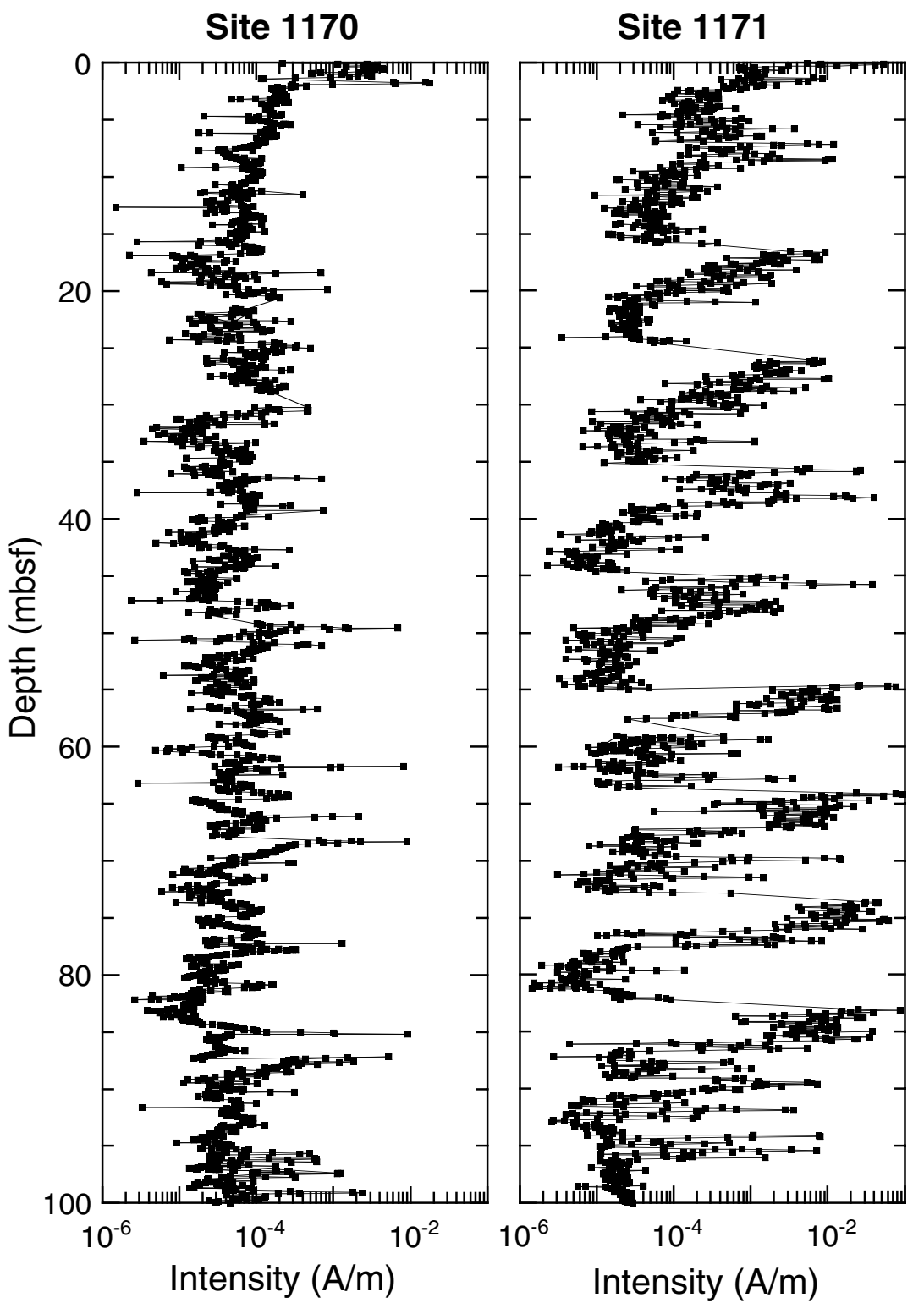


M. FULLER ET AL.

APPENDIX: MAgnetic EXPERIMENTS

Figure F6. Comparison of inclination and intensity of cores taken with standard and nonmagnetic assemblies.
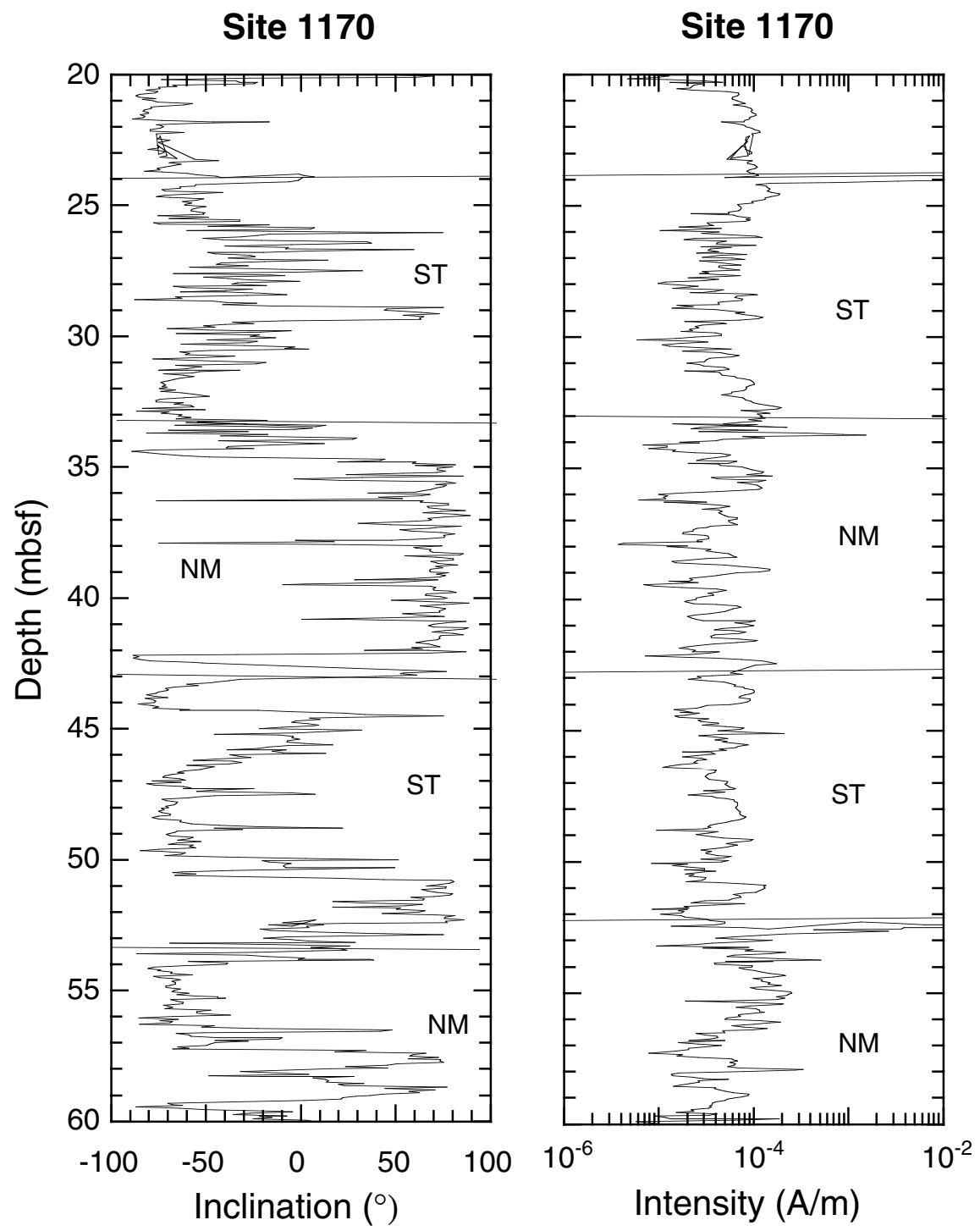